\title{
Analysis of Gas Utilization Efficiency Based on the Data of Blast Furnace Gas Center
}

\author{
Mingxin $\mathrm{Li}^{1}$, a, Lin Shi ${ }^{1}$, Yawei Liư ${ }^{2}$, Youbin Wen ${ }^{1}$ \\ ${ }^{1}$ School of Science, University of Science and Technology of Inner Mongolia, Baotou 014010, \\ Inner Mongolia, China. \\ ${ }^{2}$ Business-Intelligence of Oriental Nations Corporation Ltd, Beijing 100102, China. \\ amingxin1581@163.com
}

\begin{abstract}
This paper focuses on the detection methods of infrared photography to acquire the central data of GroupNo.6 blast furnace throat gas flow form Baotou Steel Corp. According to the situation that the distribution of blast furnace gas flow center is difficult to reflect certain regularities and class information as well as the problems that the traditional clustering methods failing to eliminate the influences of clustering noises, the density-based algorithm DBSCAN insensitive to noise with the merits of enabling to discover the class clusters of any shapes is adopted for the first time to establish the irregular classification model eliminating the noise data influence to analyze the relationship between the distribution of gas flow center and the gas utilization rate. Concept of "gas flow center deviation" is introduced in this paper. According to the research results, the gas utilization rate and the non-deviation of the gas flow center are positively correlated; the marginal development gas utilization rate is the lowest; the distribution of gas flow center presents the synchronous development type, which means the gas utilization rate is the highest when occupancy rate of gas with non-deviation and minor deviation is close to the sum and above $94 \%$. Methods adopted in this paper could rapidly identify the relationship between the gas utilization rate and the gas distribution of the gas flow center, so as to provide further evidence for the online control of the blast furnace conditions.
\end{abstract}

Keywords: furnace condition judgment; infrared photography; gas flow distribution; density-based clustering.

\section{Introduction}

The gas flow distribution is related to the furnace temperature distribution, smooth running of furnace conditions and utilization conditions of gas and finally influences the smelting indexes of blast furnace. The blast furnace operation is also carried out focusing on obtaining the reasonable and appropriate gas flow distribution. On the other hand, the gas flow distribution is also an important evidence for the blast furnace operator to judge the furnace conditions[1-4]. The purpose of this paper is to establish the clustering model, explore the relationship between the gas flow distribution pattern and gas utilization rate to realize the effective online control of blast furnace conditions.

In the recent years, various clustering algorithms are applied to the classification analysis on the distribution data of blast furnace gas flow center; however, these traditional clustering algorithms, for example, circular clustering algorithms like the hierarchical clustering and K-means clustering have failed to eliminate the noise influence[5], so that the classification data cannot reflect certain regularities, not mention explore the relationship between the classification information and gas utilization rate[4].

DBSCAN algorithm is the density-based clustering algorithm in the clustering analysis to classify the regions with enough high density into one category, discover the clusters of any shapes in the noisy space database with rapid clustering speed[6]. For the distribution of blast furnace throat gas flow center, this paper takes the $2500 \mathrm{~m} 3$ blast furnace of Baotou Steel Corp. As the research subject and makes use of the MATLAB image processing tool to make batch processing on the infrared images on the basis of infrared videos collected in one month (720) online by the infrared camera, so as to obtain the gray level image (3600 frames) within an hour. By means of statistical quantitative processing in combination with the cross temperature measurement data, the density cluster-based 
DBSCAN algorithm is adopted to obtain the clustering model. The internal relations between the classification data and gas utilization rate shall be counted and analyzed to confirm their relationship.

\section{Data Detection of the Blast Furnace Gas Flow Center}

The distribution of blast furnace throat gas flow center can be obtained by means of cross temperature measurement and infrared image detection[7-9]. This paper makes batch processing on the collected infrared videos of blast furnace and the cross temperature measurement data[10], so as to obtain the basic data of the dynamic distribution of the different gas flow centers as well as the gas utilization rates in the corresponding times. The specific process is as below:

(1)The infrared images are obtained by carrying out batch processing on the infrared videos.

(2)The feature extraction shall be carried out on each frame of infrared image to confirm the position of gas flow center in the image. (3)According to the cross temperature measurement, the thermal couple position confirms the position relation between the infrared images and actual charge level by means of space calibration. (4)The placement distribution of different gas flow centers on the images shall be abstracted. (5)Finally, the gas utilization rate within each corresponding hour shall be combined. In this paper, it has obtained 659 groups of data in total, each group of data include four indexes: occupancy rate of non-deviation gas, occupancy rate of minor-deviation gas, occupancy rate of gas with larger deviation and gas utilization rate (part of the data are as shown in Table 1)

Table. 1 Gas flow center data

\begin{tabular}{|c|c|c|c|c|}
\hline Group number & Center no offset & Center offset smaller & Center offset larger & Gas utilization rate \\
\hline 1 & $21.8 \%$ & $64.6 \%$ & $13.4 \%$ & $37.4 \%$ \\
\hline 2 & $32.9 \%$ & $52.8 \%$ & $14.1 \%$ & $39.2 \%$ \\
\hline 3 & $34.6 \%$ & $55.3 \%$ & $9.7 \%$ & $39.3 \%$ \\
\hline
\end{tabular}

\section{Density Clustering}

\subsection{Density-Based Clustering Overview}

The major ideas of density-based clustering are: to select one of the subjects as the core subject, query the adjacent domain of the core subject; once the density of the adjacent domain is exceeding a certain threshold value, any subject except for the core subject within the adjacent domain shall be selected as the core subject for the following clustering; in the end, areas with relatively higher density shall be cut by areas with relatively lower density to form clusters.

The density-based clustering algorithm can be divided into two kinds respectively based on the local connection and density function. The former is to connect the areas with relatively higher density within the local range to form a cluster, the representative algorithms include DBSCAN algorithm, OPTICS algorithm and CLIQUE algorithm etc. The latter is to simulate the data set distribution through the density function, the representative algorithms include DENCLUE algorithm etc. In this paper the most stable DBSCAN algorithm is adopted.

\subsection{DBSCAN Algorithm}

DBSCAN algorithm is a widely applied density-based clustering algorithm. It has the advantages of discovering clusters of any shapes with favorable effects of eliminating noise data. Therefore, it is well applied in various application fields. Here now we mainly introduce the DBSCAN clustering algorithm.

The algorithm idea of DBSCAN clustering algorithm is: to start from the arbitrary point $\mathrm{P}$ in the data set $\mathrm{D}$ and search for all points in $\mathrm{D}$ about $\varepsilon$ and can be reached from the density of point $\mathrm{P}$. If point $\mathrm{P}$ is the core point, so that all points in its adjacent domain and point $\mathrm{P}$ shall belong to a same cluster; these points will be regarded as the subject of the next round of observation. By continuously searching for the points that can be reached from the density of seed point to expand the cluster where they are, until a complete cluster is discovered. If point $\mathrm{P}$ is not the core point, which means there is 
no subject that can be reached from the density of point $\mathrm{P}$, so that the point $\mathrm{P}$ shall be temporarily marked as noise. Afterwards the algorithm shall repeat the above process on the next subject in D......After all seed points have been observed, a cluster is also expanded. If there are still unprocessed points in $\mathrm{D}$, the algorithm will expand the other cluster; otherwise points in $\mathrm{D}$ belonging to no cluster will be noise[6].

The algorithm will judge whether it is the core point through the $\varepsilon$ adjacent domain of each point in $\mathrm{D}$, so as to decide how to expand the cluster. Therefore, a large part of work in this algorithm is to make the area query, which means to query all points in the $\varepsilon$ adjacent domain of each point. Assuming that there are $\mathrm{n}$ points in the data set $\mathrm{D}$, without index, the entire data set will be scanned when the algorithm carries out the area query on each point in the data set.

Assuming the parameters $\varepsilon$ and MinPts are input, the DBSCAN algorithm is as below:

Step 1: to select any point $P$ from the data set and make area query on it;

Step 2: if $\mathrm{P}$ is the core point, search for all points can be reached from the density of $\mathrm{P}$ to form a cluster containing $\mathrm{P}$;

Step 3: if $\mathrm{P}$ is not the core point, $\mathrm{P}$ will be temporarily marked as the noise point;

Step 4: access the next point in the data set, repeat the above process until all points in the data set are processed.

\section{Clustering Analysis of Group No.6 Blast Furnace Gas Flow Distribution form Baotou Steel Corp.}

\subsection{Preliminary Analysis of Sample Data}

We extract 659 groups of the data and select the first three indexes of each group of data to make the clustering analysis. The three indexes are in turn the non-deviation gas occupancy rate, the smaller gas occupancy rate, and the larger gas occupancy rate. The range of the three indexes is between 0 and 1 .

Check the correlation between the data indexes. The preliminary conclusion can be made from Figure 1 that: the gas utilization rate is under the larger positive correlation influence of the nondeviation gas occupancy rate, smaller negative influence of the smaller gas occupancy rate and larger negative correlation influence of the larger gas occupancy rate.

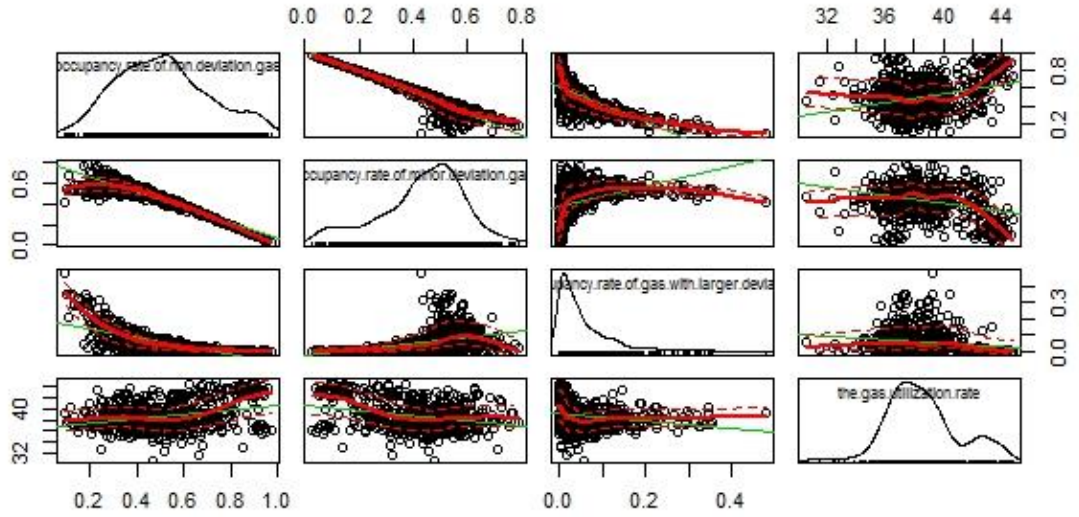

Figure .1 Four index fitting diagram

\subsection{Clustering Algorithm-Based Data Processing}

\subsubsection{Confirmation of Class Number}

According to the data and actual demand, it is inappropriate to be many classes. However the too few classes are not representative; so that 3-6 classes are selected here to compare the results of two clusters from different classes. The most suitable class number of clusters shall be selected through comparison. 
Carrying out the variance test on the clustering results of the data respectively in the two kinds of clustering methods namely the density-based cluster and K-means cluster; the output results are as shown in Table 2:

Table. 2 K-means clustering and quantitative classification based on Density Clustering

\begin{tabular}{ccccc}
\hline \multirow{2}{*}{$\begin{array}{c}\text { Category } \\
\text { quantity }\end{array}$} & \multicolumn{2}{c}{ K-means clustering } & \multicolumn{2}{c}{ Density clustering } \\
\cline { 2 - 5 } & variance & $\begin{array}{c}\text { Between class } \\
\text { variance }\end{array}$ & Within class & variance \\
\hline $\mathbf{3}$ & 563.3 & 1655.1 & 381.4 & $\begin{array}{c}\text { Between class } \\
\text { variance }\end{array}$ \\
\hline $\mathbf{4}$ & 465.3 & 1807.4 & 259.5 & 2584.7 \\
\hline $\mathbf{5}$ & 437.1 & 1976.5 & 241.2 & 222.8 \\
\hline $\mathbf{6}$ & 410.1 & 2049.5 & 226.8 & 2447.5 \\
\hline
\end{tabular}

By analyzing data in Table 2, it can be seen that the density-based cluster has better clustering effects: as it has eliminated the interference of noise data, the density-based cluster has better intraclass agglomeration and inter-class separation in comparison with $\mathrm{K}$-means. However for the classification number, when there are four classes, the intra-class variance is larger, so the clustering number is selected as four.

\subsubsection{Clustering Analysis of Empirical Data}

This paper adopts 659 groups of blast furnace gas flow center data to carry out the two kinds of clustering analysis (K-means clustering and density-based clustering) with the class number of four, so as to draw the image of clustering results. In Figure 2, the left side is the K-means clustering results, the right side is the results of density clustering based on the DBSCAN algorithm. According to the results: as it fails to eliminate the noise data, the K-means clustering is rather fuzzy while the classes are mostly crossed; as it has obviously eliminated the noise data, the density clustering has closer intra-class clustering and more obvious inter-class separation.

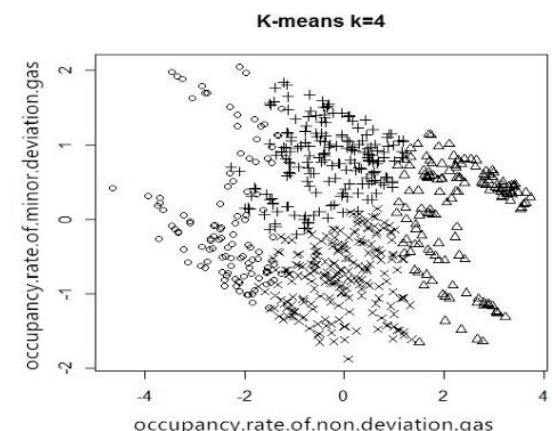

(a) K-means clustering

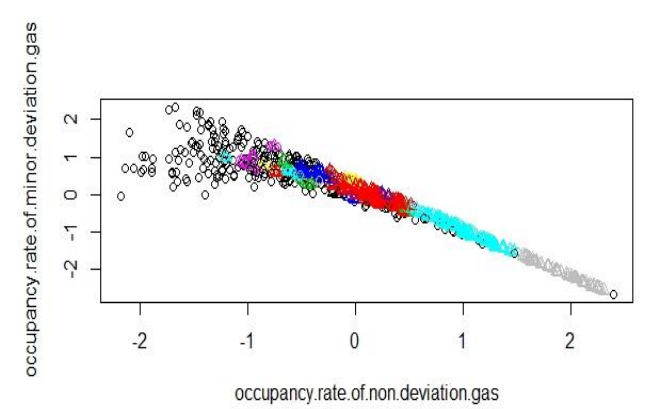

(b) density based clustering

Figure. 2 Clustering result by K-means clustering and density based clustering

\subsection{Analysis of Clustering Results}

The classification results obtained by processing the data in two kinds of clustering methods are not completely the same; however they are still very close in rough with a coincidence rate of $82 \%$. We adopt the results of density clustering to analyze each class and calculate the mean value of the occupancy rates of non-deviation gas, small-deviation gas and large-deviation gas corresponding to each class as well as the mean value of the corresponding gas utilization rate. The results are summarized as in Table 3:

Table. 3 Mean classification results by Density clustering

\begin{tabular}{ccccc}
\hline \multirow{2}{*}{ classif-ication } & \multicolumn{4}{c}{ Class mean } \\
\cline { 2 - 5 } & $\begin{array}{c}\text { No offset gas } \\
\text { occupancy }\end{array}$ & Offset smaller gas occupation & $\begin{array}{c}\text { Offset greater } \\
\text { gas occupation }\end{array}$ & Gas utilization rate \\
$\mathbf{2}$ & $11.0 \%$ & rate & $22.9 \%$ & $36.8 \%$ \\
$\mathbf{1}$ & $19.5 \%$ & $51.9 \%$ & $28.4 \%$ & $38.1 \%$ \\
$\mathbf{4}$ & $29.4 \%$ & $57.8 \%$ & $12.6 \%$ & $38.5 \%$ \\
\hline
\end{tabular}


The relationship between the gas utilization rate and the gas flow center distribution can be better explained by analyzing Table 3. (1) It can be seen by comparing the fourth class with the first, second and third classes that the gas utilization rate is positively correlated to the non-deviation of the gas flow center, which means the smaller the gas flow center deviation, the higher the gas utilization rate; what's more, it also means that the gas utilization degree is mainly the utilization rate of the central gas. (2)The gas utilization rate of marginal development is the lowest, while the occupancy rates of the second and third classes of non-deviation gases are lower and the occupancy rate of the largedeviation gas is higher. The synchronous utilization rate is also lower, which means during the blast furnace operation at this time, the gas utilization rate is determined by the central development and marginal development and dominated by the marginal development. (3)The synchronous development gas utilization rate is the highest; the occupancy rates of the fourth class of non-deviation gas and the small-deviation gas are closer to each other. The occupancy rate of the large-deviation gas is lower, which means during the blast furnace operation, the gas utilization rate is determined by the synchronous development and the gas utilization rate is the highest among the four classes.

\section{Conclusion}

This paper takes the gas flow center distribution and gas utilization rate of Group No.6 blast furnace form Baotou Steel Corp as the research subject, makes use of the 659 groups of gas flow center distribution and gas utilization rate data collected on the basis of infrared image data processing to introduce the concept of "gas flow center deviation". It establishes the density-based clustering model through the three indexes namely the occupancy rate of non-deviation gas, occupancy rate of small-deviation gas and occupancy rate of large-deviation gas. The following conclusions are made according to the clustering results in combination with the mean gas utilization rate of the clustering classes:

(1) The gas utilization rate is positively correlated to the non-deviation degree of the gas flow center, which means, the smaller the gas flow center deviation, the higher the gas utilization rate. In addition, it also means that the gas utilization degree is mainly the utilization rate of the central gas.

(2) The gas utilization rate of the marginal development is the lowest; when the occupancy rate of the non-deviation gas is lower and the occupancy rate of the large-deviation gas is higher, the gas utilization rate will be lower as well, which means during the blast furnace operation, the gas utilization rate is commonly decided by the central development and marginal development and dominated by the marginal development.

(3) The synchronous development gas utilization rate is the highest; when the occupancy rates of the non-deviation gas and the small-deviation gas are close to each other, and the sum of them occupies a higher proportion, it means that during the blast furnace operation, the gas utilization rate is determined by the synchronous development and the gas utilization rate is the highest.

(4) Regarding each blast furnace as a production individual, during the production, the gas flow center deviation and deviation position monitored on line are processed on the basis of the infrared images. Afterwards, the distribution of furnace throat gas flow shall be improved by guiding the burden distribution according to the conclusion of this paper, so as to improve the gas utilization rate and finally realize the visualized operation and online control for the burden distribution of the blast furnace.

\section{Acknowledgements}

Item Sponsored by National Natural Science Foundation of Inner Mongolia China (2017MS (LH) 0104), Innovation Fund Project of University of Science and Technology of Inner Mongolia (2015QDL17). 


\section{References}

[1]. X.F. Dong, D. Pinson, S.J. Zhang, Gas-powder flow in blast furnace with different shapes of cohesive zone, Applied Mathematical Modeling 11, (30), 1293-1309 (2006).

[2]. N. Mats, S. Henrik, Prediction of a blast furnace burden distribution variable, ISIJ International 36, (9), 1142-1150 (1996).

[3]. Y. OTSUKA, M. KONISHI, K. HANAOKA, T. MAKI, Forecasting Heat Levels in Blast Furnace Using a Neural Network Model, ISIJ Int- ernation 39, (10), 1047-1052 (1999).

[4]. J. Jimenez, J. Mochon, J.S. de Ayala, Mathematical Model of Gas Flow Distribution in a Scale Model of a Blast Furnace Shaft, ISIJ International 44, (3), 518-526 (2004).

[5]. Q. Zhu, C.L. Lu, Burden Distribution Calculation of Bell-Less Top of Blast Furnace Based on Multi-Radar Data, International Journal of Iron and steel Research 20, (6), 33-37 (2013).

[6]. X.F. Dong, A.B. Yu, J.I. YAGI, Modelling of multiphase flow in a blast furnace: recent developments and future work, ISIJ International 47, (11), 1553-1570 (2007).

[7]. M. Chu, X. Yang, F. Shen, Numerical simulartion of innovative operation of blast furnace based on multifluid model, International Journal of Iron and Steel Research 13, (6), 8-15 (2006).

[8]. K.K. Minu, M.C. Lineesh, C.J. John, Wavelet neural networks for nonlinear time series analysis, Applied Mathematical Sciences 4, (50), 2485-2495 (2010).

[9]. Y.F. Zhao, C. Jerry, Capo, Development of Burden Distribution Technology at U.S. Steel Canada's Hamilton Works 'E' Blast furnace, Iron \& Steel Technology 8, (1), 52-61 (2011).

[10]. L. Shi, Y.B. Wen, G.S. Zhao, T. Yu, Recognition of Blast Furnace Gas Flow Center Distribution Based on Infrared Image Processing, International Journal of Iron and Steel Research 23, (03), 203-209 (2016). 